\title{
Free Tissue Reconstruction of the Scalp
}

\author{
Mofiyinfolu Sokoya, MD ${ }^{1}$ Emily Misch, MD, MPH ${ }^{2}$ Aurora Vincent, MD 3 Weitao Wang, MD \\ Sameep Kadakia, MD ${ }^{5}$ Yadranko Ducic, MD, FRCS(C), FACS ${ }^{1}$ Jesse Smith, MD ${ }^{1}$
}

${ }^{1}$ Otolaryngology and Facial Plastic Surgery Associates, Fort Worth, Texas
2 Department of Otolaryngology, University of Colorado School of
Medicine, Aurora, Colorado
${ }^{3}$ Otolaryngology, Head and Neck Surgery, Madigan Army Medical
Center, Tacoma, Washington
${ }^{4}$ Department of Otolaryngology, University of Rochester Medical
Center, Rochester, New York
5 Division of Otolaryngology-Head and Neck Surgery, Department of
Surgery, Wright State University, Dayton, Ohio

Address for correspondence Yadranko Ducic, MD, FRCS(C), FACS, Otolaryngology and Facial Plastic Surgery Associates, Fort Worth, TX (e-mail: yducic@sbcglobal.net).

Semin Plast Surg 2019;33:67-71.

\begin{abstract}
Keywords

- scalp

- free flap

- reconstruction

Reconstruction of scalp defects can be accomplished by many methods, but larger defects, especially those in which the periosteum is absent or calvarial defects are present, require free tissue transfer. Various methods of scalp reconstruction, as guided by the defect components and size, are presented herein, with a focus on free tissue transfer. Different free flaps for scalp reconstructed are described with a comparison of their advantages and disadvantages. Overall, free tissue transfer for scalp defects provides a reliable, durable, and cosmetically adequate reconstructive option.
\end{abstract}

Large scalp defects can have any number of etiologies; neoplasms (commonly skin cancer) and trauma are the most common, but burns, infections (including osteomyelitis), and osteoradionecrosis can also cause defects in one or more layers of the scalp and underlying bone. ${ }^{1,2}$ Reconstruction of scalp defects can be accomplished by primary closure or with local flaps in some cases, but larger defects, especially those in which the periosteum is absent, require free tissue transfer. Various methods of scalp reconstruction, as guided by the defect components and size, are presented below.

\section{Anatomy}

Comprehensive knowledge of the scalp anatomy is essential in reconstructive surgical planning. The cranium is covered, from deep to superficial, with periosteum, loose areolar tissue, the galea aponeurotica, subcutaneous tissue, and skin. The skin of the scalp is the thickest in the body, ranging from 3 to $8 \mathrm{~mm}$ in thickness. ${ }^{3}$ Blood vessels and nerves that vascularize and innervate the skin run through the subcutaneous tissue, superficial to the galea aponeurotica. The galea is a layer of inelastic tissue that provides strength to the overlying skin and lies in continuity with the frontalis muscle fascia, occipitalis muscle fascia, and temporoparietal fascia. The galea fuses with the pericranium at the linea temporalis and conjoint tendon. The loose areolar tissue provides a potential space between the galea and pericranium; intraoperatively, it allows easy separation of the galea and pericranium, either to complete a superficial resection while leaving vascularized pericranium intact, or to allow elevation of a pericranial flap. The periosteal layer is crucial in scalp reconstruction; when intact, conservative reconstructive options remain viable, including use of acellular dermal matrix or other synthetic materials, placement of free skin grafts, and healing by secondary intention. When the periosteum is absent, however, then vascularized free tissue transfer is often required, as other reconstructive forms can fail.

Vascular anatomy is also important, especially when considering free tissue transfer. The superficial temporal vessels can be used as a recipient artery and vein, but they are often smaller caliber vessels, making microvascular anastomosis difficult, and can be prone to spasm with potential flap compromise. The facial/superficial thyroid/lingual and internal maxillary arteries are more robust, as are the external/internal/anterior jugular and retromandibular veins, but these vessels are also significantly farther away from the scalp and may require vein grafts to connect a free flap pedicle to the recipient vessels. ${ }^{2}$
Issue Theme Free Tissue Transfer Reconstruction; Guest Editor: Yadranko Ducic, MD, FRCS(C), FACS
Copyright (c) 2019 by Thieme Medical Publishers, Inc., 333 Seventh Avenue, New York, NY 10001, USA. Tel: +1(212) 584-4662.
DOI https://doi.org/ 10.1055/s-0039-1678470. ISSN 1535-2188. 


\section{Reconstructive Options in Scalp Reconstruction}

The reconstructive ladder is helpful in approaching all scalp defects, and many methods are useful for defects of varying sizes and locations. The ideal reconstruction for patients with scalp defects is (1) simple, (2) durable, and (3) cosmetically acceptable. Further, patients and their families should be comfortable with the required timeline for various reconstructive methods and any accompanying wound care before the final result is achieved. The reconstructive surgeon should also consider whether the patient will require postoperative radiotherapy (RT), and if so, should choose a reconstructive plan that will minimize healing time (so as not to delay the onset of RT) while also providing durable healing that can safely withstand the RT insult. ${ }^{4}$

First, healing by secondary intention can be used as a definitive or temporizing measure. Secondary intention is typically a better option for patients with intact periosteum, a concave surface, and lighter skin, ${ }^{5}$ but it has been shown to lead to adequate healing in some patients without intact periosteum. ${ }^{6}$ Further, healing by secondary intention may be augmented by wound vacuums and other adjuncts.

Next, primary closure is an ideal reconstructive method when possible, but it is limited by the tightness of the galea and size of some defects. Previous authors have recommended that defects less than $3 \mathrm{~cm}$ in greatest diameter are amenable to primary closure, but larger defects may require an alternative reconstruction. ${ }^{7}$ Advancement, rotation, and transposition of local tissue can allow closure of larger defects, but it is also limited by overall defect size. Local flaps are often sufficient for closure of defects less than $50 \mathrm{~cm}^{2}$ in size when the surrounding tissue has not been devascularized by RT or prior surgery. ${ }^{8,9}$

Tissue expansion can increase the amount of locally available skin, but it requires multiple visits over several weeks for (often painful) expansion of implants before definitive wound closure. ${ }^{10}$ Skin grafting is useful to cover large defects, but it requires a bed of vascularized tissue (such as the periosteal layer) or risks extensive graft failure. Next, few pedicled regional flaps provide sufficient tissue for coverage of extensive scalp defects, and many cannot reach the apex of the scalp. Thus, for composite defects and extensive defects of the scalp, free tissue transfer may be the only viable reconstructive option.

\section{Free Flap Reconstruction}

Free tissue transfer in the reconstruction of scalp defects is indicated for large defects not amenable to simpler reconstructive options or in which simpler options have failed, for reconstruction of previously radiated tissue that has a poor baseline quality, for patients in whom postoperative RT is planned and a more durable reconstruction is necessary, for patients with full-thickness calvarial bone loss, for patients with full-thickness soft tissue defects and absent periosteum, and for patients with chronic infection and compromise of the remaining soft tissue components. ${ }^{2}$ Not all patients are candidates for free tissue transfer, however, either because of limited donor vessels near the scalp or a high perioperative risk status making an extended anesthetic unfavorable. For patients amenable to free tissue reconstruction, however, there are many advantages. It can provide an immediate reconstruction of very large defects that is durable over time, resistant to the deleterious effects of RT, and cosmetically acceptable at both the donor and reconstructive sites. Further, many series have reported on free tissue transfer reconstruction of the scalp, ${ }^{2,8,11,12}$ and, in general, flap success rates from all sources are greater than $95 \%$, and partial or total flap loss is rare, making free flap reconstruction an excellent reconstructive option for many patients.

There are a variety of free flap sources for the reconstructive surgeon to consider. A 2012 review found the latissimus dorsi (LD) free flap to be the most commonly used flap in scalp reconstruction, used in $49 \%$ of reported cases. ${ }^{13}$ The rectus abdominis (RA) free flap was the next most common, used in $17 \%$ of cases, followed by anterolateral thigh (ALT) free flap ( $14 \%$ of cases) and the radial forearm free flap ( $8 \%$ of cases). ${ }^{13}$ Other sources of free flaps that have been used in scalp reconstruction include the omentum, scapular tissue, and serratus muscle. ${ }^{14}$

\section{Latissimus Dorsi}

The LD flap has historically been highly utilized in free flap reconstruction of the scalp, and it has many advantages. First, it provides a large amount of tissue allowing it to be used in subtotal or total scalp defects. ${ }^{15}$ Next, its vascular pedicle is long with large caliber vessels, making it ideal for microvascular anastamosis. ${ }^{16}$ The LD flap can be harvested with or without its overlying skin; in scalp reconstruction, it is commonly harvested as a muscle-only flap then covered by a meshed split thickness skin graft. This method has been shown to provide excellent cosmetic results, as the muscle atrophies over time allowing the flap thickness to approach the natural thickness of the scalp in its completely healed state. ${ }^{14}$ Next, a LD flap can be combined with serratus anterior, scapular, and parascapular flaps to allow larger soft-tissue coverage, and, finally, the LD flap can be harvested compositely with rib for calvarial bone reconstruction. ${ }^{16,17}$

There are a few disadvantages of the LD flap to consider. First, a disadvantage for many surgeons is intraoperative positioning. Commonly, LD harvest requires lateral decubitus positioning of the patient which may prevent simultaneous neoplasm resection and flap harvest or necessitate intraoperative position changes and lead to longer operation (OR) times. ${ }^{14}$ For example, Horn et al noted that a two-team approach was only possible in $24 \%$ of LD flaps. ${ }^{18}$ The LD can be harvested in a supine position, however, with a bump placed under the shoulder and extension of the ipsilateral arm, and, for surgeons comfortable with this method of harvest, a two-team approach is more feasible. Next, another disadvantage includes removal of a functional muscle leading to secondary shoulder dysfunction. ${ }^{19}$ Adams et al found that $35 \%$ of patients who underwent LD flaps reported moderate shoulder weakness, and $50 \%$ noted persistent back numbness/tightness. ${ }^{20}$ A systematic review in 2014 
found that LD flap patients reported significant difficulty with sports and art activities after surgery. ${ }^{21}$ Further, shoulder weakness can be particularly disabling for wheelchair-bound patients, as they may not be able to manually push their wheelchair. ${ }^{19}$ To this end, techniques have been developed to mitigate the potential for shoulder weakness; modifications involving harvest of a partial flap or a musclesparing flap have been shown to decrease morbidity. ${ }^{17,21}$

Finally, while the cosmetic result after reconstruction with a LD flap and skin graft are often acceptable, the required skin grafting adds OR time and makes the flap initially more susceptible to damage from shearing forces. Further, LD flaps can have variable postoperative muscle atrophy ultimately affecting the cosmetic outcome and, in some cases, potentially exposing underlying hardware. ${ }^{19,22}$ Overall, however, the LD flap remains a reliable, durable, and cosmetically pleasing option for free flap scalp reconstruction.

\section{Anterolateral Thigh}

The ALT flap is rapidly gaining popularity in scalp free flap reconstruction. Although found to be the third most frequently used flap in the Sweeny et al review, ${ }^{13}$ Chang et al noted in their review that the ALT was the most common flap reported in literature after the year $2000 .^{11}$

The ALT also has many advantages. Importantly, the ALT flap is very amenable to simultaneous scalp resection and flap harvest. Due to its location on the thigh, patients may remain positioned supine with ample space for two teams to fit around each surgical site. ${ }^{19} \mathrm{~A}$ two-team approach shortens operative times. Next, the ALT is a versatile flap that can be harvested as an adipocutaneous, fasciocutaneous, or chimeric flap, ${ }^{19}$ and as such, it does not require additional skin graft coverage. The vastus lateralis muscle may be harvested with the flap, as may the tensor fascia lata, either as a free graft or an attached vascular graft to be used in dural reconstruction. ${ }^{14,23}$ Additionally, the ALT has been shown to cause minimal functional morbidity. Hanasono et al found that $8 \%$ of patients had initial leg weakness or instability at their first postoperative visit, but within 6 months this resolved, and they returned to their preoperative level of activity. This finding held even when large amounts of fascia or vastus lateralis were harvested. ${ }^{24}$ Finally, the scar from ALT harvest is easily hidden beneath clothing including shorts.

Disadvantages of the ALT flap include its bulkiness and inability to cover total scalp defects. The ALT provides significant tissue bulk, but it is in the form of thicker, less pliable tissue than that from an LD flap. A comparison of LD and ALT flaps noted the mean flap surface of ALT flaps was nearly $50 \%$ than that of LD flaps (67.0 vs. $115.8 \mathrm{~cm}^{2}$, respectively). ${ }^{18}$ Kwee et al, however, report total scalp reconstruction using bilateral ALT flaps, ${ }^{25}$ and Lamaris et al report harvesting ALT flaps with median skin island dimensions of $156 \mathrm{~cm}^{2} .{ }^{19}$ The pedicle length of ALTs flaps is comparable to that of LD flaps; however, the pedicle anatomy is more variable, making its harvest more challenging. ${ }^{14,18}$ ALT bulk can also contribute to a poorer cosmetic outcome. ${ }^{14}$ Despite these disadvantages, though, the ALT flap is becoming one of the staples of scalp reconstruction.

\section{Radial Forearm}

The radial forearm flap (RFF) is not commonly used for scalp reconstruction, with Sweeny et al finding it to account for only $8 \%$ of reported scalp reconstructions. ${ }^{13}$ More surgeons are beginning to note its potential, however, and it remains a viable option for some patients. Specifically, it has been shown to work well for smaller defects ( $<7 \mathrm{~cm}$ wide) and younger patients with fuller forearms. ${ }^{13}$ For large scalp defects, however, the RFF simply cannot provide the soft tissue coverage that an ALT or LD flap can. Regardless, the RFF has many advantages. It has a reliable pedicle anatomy making for simpler dissection and harvest, and it easily allows for a two-team concurrent resection and harvest with shorter OR times. ${ }^{13}$ The RFF also has a very long pedicle with good-caliber vessels. ${ }^{14}$ In their comparison of RFFs to other flaps in scalp reconstruction, Sweeny et al found that patients who underwent RFF reconstruction had shorter hospital stays and fewer complications than those reconstructed with other flaps. ${ }^{13}$

The primary disadvantage of the RFF is its size and its inability to cover total scalp defects. It should only be used in defects less than $200 \mathrm{~cm}^{2}$. Additionally, while donor site morbidity is low, it is not without side effects. A study by Hekner et al found that $15 \%$ of patients reported reduced strength in the donor hand. Pressure and cold perception were diminished as well. ${ }^{26}$ Further, Riecke et al found objective reductions in grip strength, pinch strength, and wrist movement in patients after RFF. ${ }^{27}$ Thus, while the RFF is not an ideal option for many patients, it should nonetheless be considered for smaller scalp defects (-Figs. 1, 2, 3).

\section{Other Flaps}

Several other flaps have been used less frequently for scalp reconstruction. The RA flap, for example, was historically commonly used for scalp reconstruction, ${ }^{28}$ though its use has

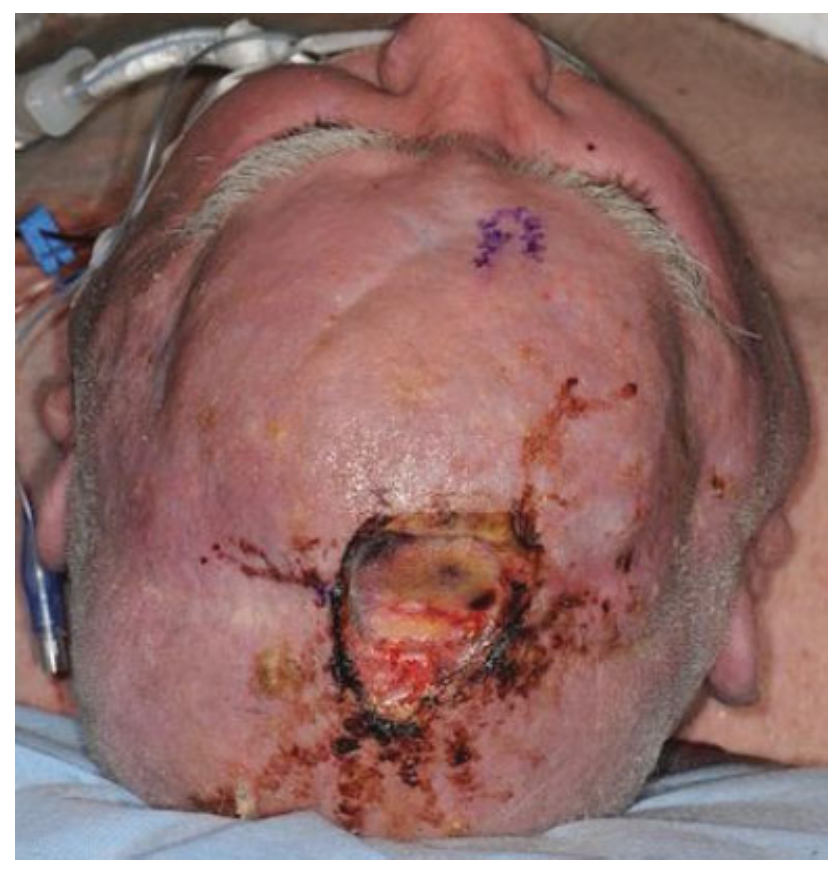

Fig. 1 Preoperative appearance of a vertex scalp defect after multiple attempts at local flap closure. 


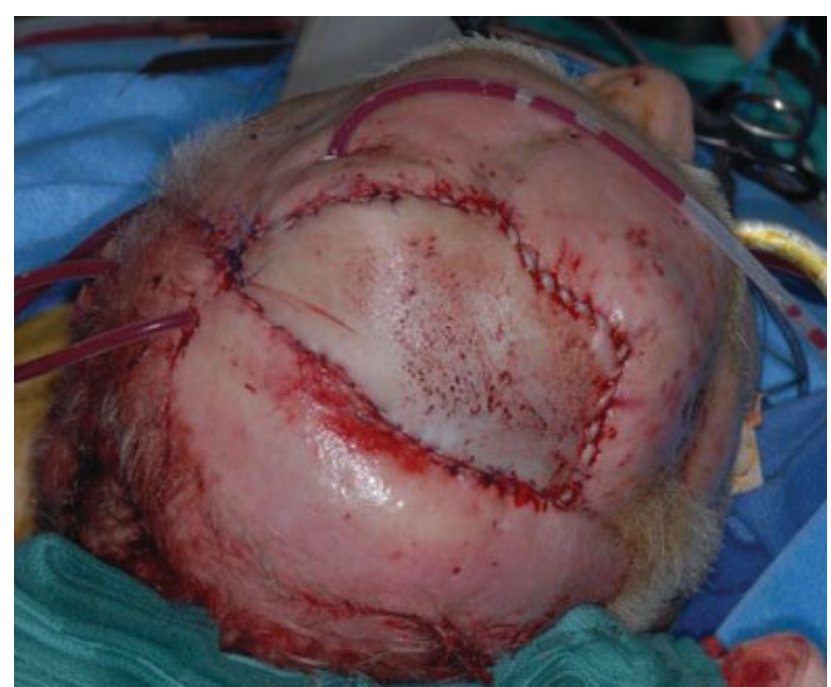

Fig. 2 Immediate postoperative appearance after microvascular free tissue reconstruction of scalp defect with radial forearm free flap.

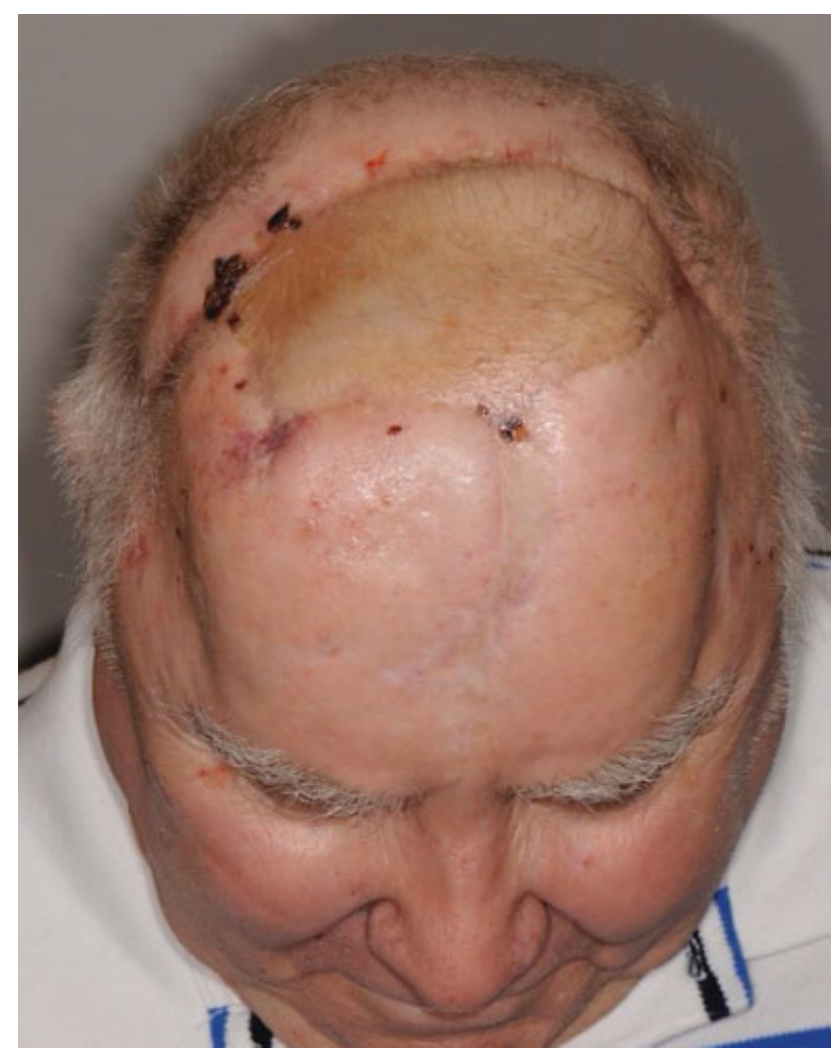

Fig. 3 Six-month postoperative appearance after radial forearm free flap.

decreased over the past two decades. Still, this flap has many advantages. First, it has a reliable anatomy allowing ease of harvest. Next, its location allows a two-team approach with simultaneous tumor extirpation and preparation of recipient vessels with flap harvest. ${ }^{29}$ The RA provides significant tissue for total scalp defect coverage, and the donor site is often amenable to primary closure with adequate cosmesis. ${ }^{29}$ Finally, the RA flap has a long vascular pedicle that can obviate the need for vein grafting to recipient vessels. ${ }^{10}$

The RA also has several disadvantages, however. First, its harvest can increase the risk of hernia formation. Next, while it provides a significant amount of soft tissue, the tissue can be bulkier and less pliable than muscle harvested with an LD flap. ${ }^{8}$

The omental free flap is another flap that has been used for subtotal and total scalp reconstruction, covered by a skin graft. ${ }^{10}$ The omental flap can provide a large amount of thin, pliable soft tissue with a very long pedicle $(>20 \mathrm{~cm})$ that can easily reach recipient vessels in the neck. ${ }^{10}$ The omental flap harvest requires laparotomy, however, and risks postoperative adhesion formation. Finally, the serratus and scapular free flaps have been successfully applied to scalp reconstruction. These flaps can provide significant and bulky soft tissue, and the scapular flap can be harvested in an osteocutaneous fashion. These flaps require prone positioning for harvest, however, which can necessitate an intraoperative position change and lengthen the procedure. Overall, other flaps have become more popular in scalp reconstruction due to ease of harvest.

\section{Conclusion}

Free tissue transfer is a reliable, durable method for reconstructing large defects of the scalp. The LD is the most commonly used flap, although the ALT and others have also been repeatedly shown to lead to successful reconstruction. When approaching a scalp defect, the reconstructive surgeon should consider not only the defect size and anatomic components, but also the patient condition and goals in identifying the ideal reconstructive plan.

\section{Financial Disclosures \\ None.}

\section{Conflict of Interest}

None.

\section{References}

1 Mueller CK, Bader RD, Ewald C, Kalff R, Schultze-Mosgau S. Scalp defect repair: a comparative analysis of different surgical techniques. Ann Plast Surg 2012;68(06):594-598

2 Steiner D, Horch RE, Eyüpoglu I, et al. Reconstruction of composite defects of the scalp and neurocranium-a treatment algorithm from local flaps to combined AV loop free flap reconstruction. World J Surg Oncol 2018;16(01):217-228

3 Shestak KC, Ramasastry SS. Reconstruction of defects of the scalp and skull. In: Cohen M, ed. Mastery of Plastic and Reconstructive Surgery. Boston, MA: Little, Brown; 1994:830-841

4 Hussussian CJ, Reece GP. Microsurgical scalp reconstruction in the patient with cancer. Plast Reconstr Surg 2002;109(06):1828-1834

5 Desai SC, Sand JP, Sharon JD, Branham G, Nussenbaum B. Scalp reconstruction: an algorithmic approach and systematic review. JAMA Facial Plast Surg 2015;17(01):56-66

6 Becker GD, Adams LA, Levin BC. Secondary intention healing of exposed scalp and forehead bone after Mohs surgery. Otolaryngol Head Neck Surg 1999;121(06):751-754

7 Raposio E, Nordström RE, Santi PL. Undermining of the scalp: quantitative effects. Plast Reconstr Surg 1998;101(05):1218-1222

8 Oh SJ, Lee J, Cha J, Jeon MK, Koh SH, Chung CH. Free-flap reconstruction of the scalp: donor selection and outcome. J Craniofac Surg 2011;22(03):974-977

9 Steiner D, Hubertus A, Arkudas A, et al. Scalp reconstruction: a 10-year retrospective study. J Craniomaxillofac Surg 2017;45(02):319-324 
10 Mehrara BJ, Disa JJ, Pusic A. Scalp reconstruction. J Surg Oncol 2006;94(06):504-508

11 Chang KP, Lai CH, Chang CH, Lin CL, Lai CS, Lin SD. Free flap options for reconstruction of complicated scalp and calvarial defects: report of a series of cases and literature review. Microsurgery 2010;30(01):13-18

12 Eck DL, Koonce SL, Al Majed BM, Perdikis G. Evaluation of options for large scalp defect reconstruction: a 12-year experience. Eplasty 2014;14:e10

13 Sweeny L, Eby B, Magnuson JS, Carroll WR, Rosenthal EL. Reconstruction of scalp defects with the radial forearm free flap. Head Neck Oncol 2012;4:21

14 Fowler NM, Futran ND. Achievements in scalp reconstruction. Curr Opin Otolaryngol Head Neck Surg 2014;22(02):127-130

15 Hierner R, van Loon J, Goffin J, van Calenbergh F. Free latissimus dorsi flap transfer for subtotal scalp and cranium defect reconstruction: report of 7 cases. Microsurgery 2007;27(05):425-428

16 Trignano E, Fallico N, Nitto A, Chen HC. The treatment of composite defect of bone and soft tissues with a combined latissimus dorsi and serratus anterior and rib free flap. Microsurgery 2013; 33(03):173-183

17 Herrera F, Buntic R, Brooks D, Buncke G, Antony AK. Microvascular approach to scalp replantation and reconstruction: a thirty-six year experience. Microsurgery 2012;32(08):591-597

18 Horn D, Jonas R, Engel M, Freier K, Hoffmann J, Freudlsperger C. A comparison of free anterolateral thigh and latissimus dorsi flaps in soft tissue reconstruction of extensive defects in the head and neck region. J Craniomaxillofac Surg 2014;42(08): 1551-1556

19 Lamaris GA, Knackstedt R, Couto RA, Abedi N, Durand P, Gastman B. The anterolateral thigh flap as the flap of choice for scalp reconstruction. J Craniofac Surg 2017;28(02):472-476
20 Adams WP Jr, Lipschitz AH, Ansari M, Kenkel JM, Rohrich RJ. Functional donor site morbidity following latissimus dorsi muscle flap transfer. Ann Plast Surg 2004;53(01):6-11

21 Lee KT, Mun GH. A systematic review of functional donor-site morbidity after latissimus dorsi muscle transfer. Plast Reconstr Surg 2014;134(02):303-314

22 Lin PY, Miguel R, Chew KY, Kuo YR, Yang JC. The role of the anterolateral thigh flap in complex defects of the scalp and cranium. Microsurgery 2014;34(01):14-19

23 Shimizu F, Oatari M, Matsuda K, Uehara M, Sato S, Kato A. Algorithm for reconstruction of composite cranial defects using the fascial component of free anterolateral thigh flaps. J Craniofac Surg 2013;24(05):1631-1635

24 Hanasono MM, Skoracki RJ, Yu P. A prospective study of donor-site morbidity after anterolateral thigh fasciocutaneous and myocutaneous free flap harvest in 220 patients. Plast Reconstr Surg 2010;125(01):209-214

25 Kwee MM, Rozen WM, Ting JW, Mirkazemi M, Leong J, Baillieu C. Total scalp reconstruction with bilateral anterolateral thigh flaps. Microsurgery 2012;32(05):393-396

26 Hekner DD, AbbinkJH, van Es RJ, Rosenberg A, Koole R, Van Cann EM. Donor-site morbidity of the radial forearm free flap versus the ulnar forearm free flap. Plast Reconstr Surg 2013;132(02):387-393

27 Riecke B, Kohlmeier C, Assaf AT, et al. Prospective biomechanical evaluation of donor site morbidity after radial forearm free flap. Br J Oral Maxillofac Surg 2016;54(02):181-186

28 Woodworth BA, Gillespie MB, Day T, Kline RM. Muscle-sparing abdominal free flaps in head and neck reconstruction. Head Neck 2006;28(09):802-807

29 Borah GL, Hidalgo DA, Wey PD. Reconstruction of extensive scalp defects with rectus free flaps. Ann Plast Surg 1995;34(03):281-285, discussion 285-287 